REVISTA DE ESTUDIOS E INVESTIGACIÓN

EN PSICOLOGÍA Y EDUCACIÓN

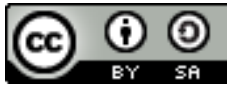

ISSN: 1138-1663; elSSN: 2386-7418

UDC / UMinho

2021, Vol. 8, No. 2, 282-300.

DOI: https://doi.org/10.17979/reipe.2021.8.2.8566

\title{
Impacto do Sars-Cov-2 (COVID-19) na cognição social e saúde mental de professores brasileiros
}

\section{Impact of Sars-Cov-2 (COVID-19) on social cognition and mental health among Brazilian teachers}

\author{
Diana Ramos-Oliveira (iD) https://orcid.org/0000-0001-7604-4825 \\ Luciana Xavier Senra (iD) https://orcid.org/0000-0002-5058-1574 \\ Programa de Pós-graduação em Psicologia, Centro de Ciências da Saúde, Universidade Católica \\ de Petrópolis: https://www4.ucp.br/ \\ Rio de Janeiro - Brasil
}

O financiamento para este estudo foi apoiado pelo Conselho Nacional de Desenvolvimento Científico e Tecnológico (CNPq) e a Fundação Celso da Rocha Miranda (FCRM/UCP) na concessão de Bolsas de Estudos de Iniciação Científica.

Correspondência relativa a este artigo: Diana Ramos-Oliveira - ramosdeoliveira.diana@gmail.com 


\title{
Resumen
}

O objetivo deste estudo foi analisar a cognição social e a saúde mental de professores brasileiros, em resposta ao COVID-19. Participaram 320 docentes de todos os níveis de ensino ( $82 \%$ mulheres), com uma média de idade de 46 anos. Para a coleta de dados foi utilizado um questionário on-line sobre a percepção da ameaça da covid-19, a percepção da ameaça ao trabalho, a saúde geral, a depressão, o impacto da covid-19 e a satisfação com a vida. Os resultados mostram que a percepção da ameaça da covid19 está correlacionada com a saúde mental em geral e com indicadores de depressão, sugerindo uma presença de desmotivação, falta de perspectivas, insônia no cotidiano dos professores. Em relação à saúde mental, os resultados mostraram correlação com a depressão e leve relação com a satisfação com a vida, sugerindo que, nas questões de autoconfiança, os professores se sentem infelizes e deprimidos. A capacidade de concentração dos professores também tem sido prejudicada pela impossibilidade de realizarem as suas actividades diárias. Finalmente, os resultados para a depressão foram significativos, embora tenha sido observada uma fraca correlação com o impacto da COVID-19; os indicadores de depressão foram também negativamente correlacionados com a satisfação de vida. $O$ artigo conclui que as queixas dos professores sobre aspectos econômicos, psicológicos e de recursos dependem do valor que atribuem a cada domínio em termos da sua importância para a satisfação com a vida.

Palabras clave: COVID-19, cognição social, saúde mental, professores, Brasil

\begin{abstract}
The aim of the study was to analyse social cognition and mental health among Brazilian teachers following the COVID-19 pandemic. The sample for the study comprised a total of 320 teachers from all levels of education ( $82 \%$ women), with an average age of 46 years. Data were collected using an online questionnaire on the perception of the threat of COVID-19, the perception of the threat to work, general health, depression, impact of COVID-19, and life satisfaction. The results showed a correlation between mental health in general and indicators of depression and the perception of the threat of COVID-19, suggesting a lack of motivation, lack of opportunities and insomnia in teachers' daily lives. Regarding mental health, the results showed a correlation with depression and a slight connection with life satisfaction, suggesting that, in matters of self-confidence, teachers feel unhappy and depressed. Teachers' ability to concentrate has also been impaired by being unable to carry out their daily activities. Finally, the results for depression were significant, although a weak correlation was observed with the impact of COVID-19; indicators of depression were also negatively correlated with life satisfaction. The article concludes that teachers' complaints about financial, psychological and resourcing issues depend on the value they place on each domain in terms of its importance to life satisfaction.
\end{abstract}

Keywords: COVID-19, social cognition, mental health, teachers, Brazil 
Em 31 de Dezembro de 2019, o mundo foi apresentado ao novo coronavírus, SARS-CoV2, e diante das proporções de contágio e elevados números de mortes em março de 2020, a Organização Mundial da Saúde (OMS) já declarava Emergência de Saúde Pública de Importância Internacional, ou seja, uma pandemia (Daniel, 2020; Ortiz, 2020;). Este contexto perturbou profundamente, entre outras áreas, os sistemas de educação em todo o planeta, mudando a aparência das salas de aula e do aprendizado cotidiano. Os educadores estão em um cenário em constante mudança, lidando com a saúde de alunos, deles enquanto professores e da comunidade em geral. Neste panorama, os educadores se expõem a diversas situações e pressões institucionais, o que reverbera no adoecimento, principalmente da saúde mental.

Para entender o contexto citado é importante referir ao aporte teórico da Cognição Social, a qual, em essência, a cognição social trata de investigar como as pessoas compreendem as outras pessoas e elas mesmas (Fiske \& Taylor, 2016). Nesta compreensão as pessoas são agentes causais que percepcionam e são percepcionadas e entre os principais elementos deste processo estão as crenças, desejos, emoções e traços de personalidade, bem como a forma e a repercussão do que percebemos em diversos comportamentos. Tais elementos são alvos difíceis de cognição porque se ajustam ao fato de serem percebidos e muitos dos seus atributos serem inferidos e passíveis de mudança (Ostrom, 1984). Nota-se que, essa característica fez do conceito quase sinônimo de percepção social, dado que compreender a personalidade, as intenções, as crenças e a identidade dos outros pode ser a forma mais importante de percepção em que os humanos se envolvem (Lieberman \& Pfeifer, 2005).

De acordo com Leyens e Dardenne (1996), no campo da percepção social, as concepções do indivíduo como percepiente, pensador e agente social têm evoluído e se modificado ao longo dos anos e, podem ser sistematizadas em cinco grandes perspectivas, apoiados em grandes teorias e evidências empíricas: a) ser racional e consistente: alguém que se apoia na racionalização e na procura de consistência para reduzir o estado de desconforto psicológico provocado pela inconsistência entre as suas cognições; b) cientista ingênuo: as pessoas processam informação, recorrendo sobretudo às suas teorias e não a um processamento mais elementar que exige o exame detalhado e objetivo da informação; c) processador de dados: que aborda os fatos de forma objetiva na ausência de preconcepções (Anderson, 1981); d) um avaro cognitivo: aludindo à relutância com que as pessoas utilizam as suas capacidades para perceber os outros de forma precisa e d) um estrategista social motivado: que dispõe de várias formas de pensar que seleciona e utiliza com base nos seus objetivos, motivos e necessidades (Fiske \& Taylor, 2016). 
No século passado, estudos sobre a percepção do isolamento social, conhecida coloquialmente como solidão, foi caracterizada nas primeiras pesquisas científicas como "sofrimento crônico" (Weiss, 1973). No século XXI, estudos sobre esta temática sugerem que a dor social da solidão evoluiu como um sinal de que as conexões dos indivíduos com os demais estão enfraquecendo, e para motivar o reparo e a manutenção delas, é necessário assegurar saúde e bem-estar, inclusive para a sobrevivência de nossos genes (Caciopo \& Patrick, 2008).

As pessoas em isolamento, em contraste com as não isoladas, estão em alerta máxima para ameaças sociais. Portanto, os indivíduos solitários tendem a ver seu mundo social como ameaçador e punitivo. Pesquisas experimentais sobre o isolamento demonstraram que a solidão não apenas faz com que as pessoas se sintam mais ansiosas, temam avaliações negativas e ajam com mais frieza em relação aos outros, mas também fazem com que se sintam mais frias (Cacciopo et al., 2006; Zhong \& Leonardelli, 2008). Ademais, tendem a formar impressões sociais mais negativas dos outros, e suas expectativas, raciocínio de atribuição e ações para com os outros tendem a ser menos generosas comparadas àquelas não solitárias. Quando as expectativas sociais negativas de um indivíduo suscitam comportamentos de outras pessoas que validam essas expectativas, estas são reforçadas e aumentam a probabilidade de o indivíduo se comportar de forma a afastar as mesmas pessoas de quem ele mais deseja estar próximo para melhor cumprir sua vida social (Caciopo \& Hawkley, 2005). Consequentemente, as pessoas isoladas podem se ver como vítimas passivas em seu mundo social, mas são contribuintes ativos por meio de suas interações autoprotetoras e paradoxalmente autodestrutivas com outras pessoas (Downey \& Romero-Canyas, 2005; Murray et al., 2003).

No contexto educativo, como demonstra Orozco-Solís et al. (2021), em seu estudo sobre as relações intrafamiliares e conexão com escola, professores e alunos, e resiliência em adolescentes em contexto de adversidade. Os autores argumentam que o sentido de ligação com a família e a escola é um elemento associado ao desenvolvimento psicossocial dos adolescentes, sendo um fator que pode influenciar a sua forma de responder às adversidades e de se ajustar às mudanças. Os resultados mostraram que o ambiente familiar é o principal fator que determina como os adolescentes enfrentam as adversidades.

A maioria das pessoas são resiliente ao estresse, e muitos sobreviventes de eventos altamente ameaçadores provavelmente sairão psicologicamente ilesos. Todavia, durante uma pandemia, muitas pessoas ficarão com medo, algumas de maneira intensa (Taylor, 2017, 2019). Isso decorre da variabilidade de resposta aos determinantes psicossociais do estresse, da percepção de ameaça ou a ocorrência real de uma pandemia. Estas respostas vão do medo à indiferença e/ou ao fatalismo (Wheaton et al., 2012). 
Fletcher et al., (2020) em pesquisa realizada no Reino Unido para explorar como as pessoas percebem a ameaça do COVID-19 à sua saúde, finanças, economia e saúde da população, constatou que a maioria das pessoas ainda percebe o COVID-19 como bastante ameaçador ou muito ameaçador para a economia do Reino Unido (94\%), para a saúde da população (80\%) e saúde pessoal (54\%); $41 \%$ afirmam que pandemia é uma ameaça às suas finanças pessoais. Ademais, evidenciou que existem diferenças na percepção da ameaça do COVID-19 aos indivíduos (sua saúde pessoal e finanças, em oposição à saúde da população e da economia) por variáveis demográficas. Apenas 39\% das pessoas com idade entre 18-34 o veem como bastante ameaçador ou muito ameaçador para a saúde pessoal, comparado aos dois terços (63\%) daquelas com 55 anos ou mais. Da mesma forma, os maiores de 55 anos têm menos probabilidade de sentir que o COVID-19 ameaça suas finanças pessoais.

O estudo sobre percepção da ameaça, COVID-19 e altruísmo realizado por Vieira et.al., (2020) nos Estados Unidos revelaram em dimensão populacional, que não houve indicação de mudança nos níveis de altruísmo ao longo da pandemia. No âmbito individual, entretanto, a percepção da ameaça de COVID-19 foi associada ao aumento do altruísmo diário. Os autores salientam haver uma associação robusta entre experimentar ansiedade aguda e alta excitação fisiológica durante a pandemia (característica de maior iminência de ameaça percebida) e o envolvimento em comportamentos altruístas. Essas descobertas apoiam uma relação entre a motivação defensiva e altruísta em humanos e sugerem que a relação pode ser facilitada pela iminência da ameaça (Vieira et al., 2020). Para conhecer os impactos psicossociais da COVID-19, um estudo de revisão encontrou que, a própria doença multiplicada pela quarentena forçada para combater COVID-19, aplicada por bloqueios nacionais, pode produzir pânico agudo, ansiedade, comportamentos obsessivos, acumulação, paranoia, depressão e transtorno de estresse pós-traumático (PTSD) a longo prazo (Dubey et al., 2020).

A pandemia por COVID-19 e o ambiente educativo se tornaram um tema importante nas discussões atuais. Entretanto, observa-se, até então, pesquisas sobre os efeitos na saúde mental dos professores no Brasil é deficitário. Neste contexto, a figura do professor, antes peça fundamental no ensino-aprendizagem, agora passa ser como um mero figurante neste cenário de caos nacional ou porque não dizer mundial (Muñoz, 2020). Alguns autores argumentam que os professores acabam adoecendo por não alcançar os objetivos indicados pelas instituições e pelas pressões relacionadas ao uso das tecnologias (Silva et al., 2020). Pesquisas sugerem que as tecnologias digitais precisam transcender a educação bancária de difusão de conhecimentos, devendo ser criados espaços de participação, reflexão dialógica, desenvolvendo o raciocínio clínico, crítico e diferenciado (McKimm et al., 2020). 
No contexto brasileiro, dentre os escassos trabalhos encontrados sobre os docentes no período da pandemia por COVID-19, encontra-se a pesquisa que identifica aspectos emocionais de estudantes e professores de uma instituição pública do Estado de São Paulo em relação a reorganização do calendário escolar com aulas na modalidade de Ensino a Distância em época de isolamento social devido à pandemia de COVID-19 que assola o Brasil. Os resultados mostraram estudantes e professores apresentando emoções distintas, frustração, medo e a tristeza, são as emoções que mais se destacam (Reis et al., 2020).

O confinamento no segundo semestre letivo de 2020 mudou a rotina de todos os professores, principalmente aqueles que, em sua maioria ministravam disciplinas presenciais. Argumentam que se adaptar a esta nova rotina de home-office tem sido um desafio constante em meio a tantos sentimentos experienciados, tais como: a ansiedade, a desmotivação, o desânimo, a sobrecarga de atividades, as mudanças de estratégias, dentre outras (Haubrich \& Froehlich, 2020). Em consonância com esses dados, estudos demonstraram que o adoecimento docente é expresso pelas incertezas, estresses, ansiedade e depressão, e tem conduzido à síndrome do esgotamento profissional (Araújo et al., 2020).

No cenário internacional, encontra-se pesquisas sobre a relação do docente, a saúde mental e a COVID-19. Entre estas, o estudo realizado na China, revelando que inúmeros docentes apresentam problemas na saúde mental pela COVID-19, como o transtorno depressivo leve, transtorno afetivo bipolar, ansiedade generalizada, transtorno de adaptação e síndrome de Burnout ou síndrome do esgotamento profissional (Wang \& Wang, 2020). Nesse sentido, existe argumento que esse contexto revela que os docentes universitários estão inseridos em um ambiente propício ao adoecimento mental pelos impactos da COVID-19, seja pelas notícias jornalísticas de morbimortalidade, seja pelas pressões oriundas das instituições de ensino superior relacionadas ao uso das tecnologias digitais, somadas a vida conjugal, materna e doméstica e tantas outras atribuições que lhes são conferidas (Shaw, 2020).

Neste contexto, o estudo visou examinar a relação entre a percepção social da ameaça e a saúde mental em resposta a pandemia por COVID-19 dos professores brasileiros. Trata-se de pesquisa transversal de caráter exploratória, quantitativa correlacional, realizada com docentes brasileiros que estiveram e/ou estão lecionando remotamente durante a pandemia por COVID-19.

\section{Método}

\section{Participantes}

A amostra não probabilística que envolveu $N=320$ professores do Sistema Educacional Brasileiro, a maioria mulheres $81.9 \%$ com idades entre 35 e 50 anos $(M=45.9, D P=9.80)$, e 
majoritariamente inseridos no ensino Fundamental (59.1\%), seguido do Ensino médio 27.5\% e do Ensino Superior 23.8\% (Ver Tabela 1). Os 65\% são funcionários de Escolas ou Universidades públicas e $27.5 \%$ com contratos. Durante a pandemia, $80.9 \%$ trabalham em home office.

\section{Tabela 1}

Professores segundo Segmento Educacional que atuam

\begin{tabular}{lccc}
\hline Segmento Educacional & $f(\%)$ & Segmento Educacional & $f(\%)$ \\
\hline Educação Infantil & $17.2(55)$ & Ensino Médio & $27.5(88)$ \\
Pré-escola & $5.6(18)$ & Ensino Médio Técnico & $5.3(17)$ \\
Ensino Fundamental & $59.1(189)$ & Ensino Superior & $23.8(76)$ \\
Ensino no Campo & $0.3(1)$ & Educação de Jovens e Adultos - EJA & $12.5(40)$ \\
Educação Especial & $5.0(16)$ & & \\
\hline
\end{tabular}

\section{Instrumentos}

Percepção de Ameaça do Coronavírus (Conway et al., 2020): Avalia os quão ameaçados ou preocupados estão com o COVID-19. A escala unifatorial na versão abreviada apresenta excelente propriedades psicométricas no estudo original $\alpha=.90$. Consiste em seis itens tipo Likert de 7 pontos, em que 1-Discordo Totalmente e 7-Concordo Totalmente. O CFI = 0.98, e o Índice de Tucker-Lewis $(T L I)=0.97$, ultrapassou os mínimos normalmente aceitos $(0.90)$ e os padrões normalmente aceitos para um "bom" ajuste da medida aos dados (.95; Lai \& Green, 2016; Schreiber et al., 2006). Conforme indicado pelo RMSEA $=0.09$ (IC 90\% $=0.05,0.13, p=.04)$ e SRMR = 0.03. Os padrões típicos para RMSEAs e SRMRs adequados são 0.10 e abaixo, com 0.05 e abaixo indicando um "bom" ajuste (Lai \& Green, 2016). No geral, este modelo tem bom ajuste relativo, conforme indicado pelo CFI e TLI), e ajuste absoluto (conforme indicado pelo RMSEA e SRMR).

Percepção Social da Ameaça ao Trabalho: Este instrumento foi desenvolvido para este estudo, sem o intuito de realizar validação psicométrica, mas baseado no instrumento de Conway et al. (2020) relacionado ao COVID-19, com oito itens que se referem ao trabalho, as aulas e a quarentena em família durante a pandemia (como: Estou satisfeito em trabalhar em home office; Sua instituição de ensino respeita sua privacidade; Me incomoda ter tantas pessoas em casa comigo na quarentena), os quais são respondidos em uma escala Likert de 7 pontos (1 $=$ Discordo Totalmente e 7 = Concordo Totalmente). Os scores são obtidos por meio da soma das respostas; quanto maior a soma, maior será a percepção social de ameaça ao trabalho.

Questionário de Saúde Geral (QSG-12): Versão abreviada do Questionário de Saúde Geral de Goldberg (1972), adaptado para o Brasil por Pasquali et al. (1994) revisada por Gouveia, 
Chaves et al. (2003) (com modelo unifatorial e $\alpha=.84$ ), que expressa um índice geral da saúde mental. O instrumento se compõe de 12 itens, cada item é respondido em termos do quanto a pessoa tem experimentado os sintomas descritos, evidenciados em escala de quatro pontos. No caso dos itens que negam a saúde mental (por exemplo: Suas preocupações lhe têm feito perder muito sono? ou Tem se sentido pouco feliz e deprimido?), as alternativas de resposta variam de 1 (Absolutamente, não) a 4 (Muito mais que de costume); no caso dos itens afirmativos (por exemplo: Tem se sentido capaz de tomar decisões? ou Tem podido concentrar-se bem no que faz?), as respostas foram de 1 (Mais que de costume) a 4 (Muito menos que de costume). A apuração exige inversão dos itens negativos, isto é, quanto menor o escore, melhor a condição de saúde mental.

Patient Questionaire Health (PHQ-9) (Santos et al., 2013). Avalia a presença de sintomas para episódio de depressão maior, conforme DSM-V. Constitui nove perguntas referentes a sintomas indicativos de humor deprimido, anedonia (perda de interesse ou prazer em fazer as coisas), problemas com o sono, cansaço ou falta de energia, mudança no apetite ou peso, sentimento de culpa ou inutilidade, problemas de concentração, sentir-se lento ou inquieto e pensamentos suicidas. A frequência de cada sintoma nas últimas duas semanas é avaliada em escala Likert em que 0 = Nenhum dia, 1 = Menos de uma semana, $2=$ Uma semana ou mais e 3 = Quase todos os dias. O questionário ainda inclui uma décima pergunta que avalia a interferência desses sintomas no desempenho de atividades diárias, como trabalhar e estudar. A classificação se dá nos níveis em ausente (de 0 a 4), leve (entre 5 e 9), moderada (entre 10 e 14), moderadamente severa (entre 15 e 19) e severa (entre 20 e 27) (Kroenke, et al., 2001; Spitzer et al., 1999).

Questionário sobre o Impacto COVID-19 (Conway et al. (2020): Foi usada a escala que avalia impactos na vida do indivíduo relacionados ao COVID-19. A versão é composta por sis itens com formato Likert de 7 pontos, quando $1=$ Discordo Totalmente e 7 = Concordo Totalmente. Apresenta três subescalas: impacto financeiro, impacto nos recursos e impacto psicológico.

Satisfação com a vida (Diener et al., 1985): Validada no Brasil por Gouveia et.al. (2005), é uma escala unifatorial, que visa avaliar um componente cognitivo do bem-estar subjetivo, o julgamento que as pessoas fazem acerca do quanto estão satisfeitas com suas vidas. Como os indivíduos provavelmente dão pesos e significados diferentes a domínios específicos de suas vidas. Compõe-se de cinco itens de natureza global respondidos em uma escala Likert, variando de 1 (Discordo Totalmente) a 7 (Concordo Totalmente). Para a correção da escala, deve-se somar 
todos os itens respondidos, obtendo-se o escore bruto sendo que quanto mais alta a pontuação, maior é a satisfação com a vida.

\section{Procedimentos}

Os participantes foram convidados através de divulgações por meio das redes sociais, assim como através de contatos com docentes em várias partes do país, em que o link foi disponibilizado pela plataforma on-line Survey Monkey, para coleta dos dados. Primeiramente, tiveram acesso à um convite explicando os objetivos da pesquisa, em seguida estiveram cientes sobre a pesquisa e assinaram o Termo de Consentimento Livre e Esclarecido (TCLE) on-line garantindo o sigilo e anonimato.

O referido trabalho recorreu-se as diretrizes e normas regulamentadoras estabelecidas na Resolução do Conselho Nacional de Saúde (CNS) 510/2016, que estabelece as regras aplicáveis à pesquisa em Ciências Humanas e Sociais, não sendo registrada ou avaliada pelo Comitê de Ética e Pesquisa / Conselho Nacional de Ética e Pesquisa, garantindo o cumprimento dos fundamentos éticos e científicos da resolução (que assegura a pesquisa de opinião pública com participantes não identificados).

\section{Resultados}

Na Tabela 2 são apresentadas as médias das variáveis tratadas neste estudo. Inicialmente se destaca como os professores sentem o impacto da COVID-19 $(\alpha=.83)(M=34.99, D P=12.09)$, quer seja em aspectos financeiros ou psicológicos. Foi realizado uma análise fatorial com os dados obtidos, tendo observado ser um modelo bifatorial explicando $41.53 \%$ da variância total, apresentando um alfa de Cronbach $=.57$ para a percepção social de ameaça ao trabalho $(M=$ $4.21, D P=.947)$ relacionados a queixas da pressão das instituições, ao trabalho em home office e o sentir-se mal em ter que dar aulas ao vivo, corroborando com outros estudos, por exemplo Haubrich e Froehlich (2020) que revelam a fala dos professores em ter que se adaptar à nova rotina de home office e os desafios que supõe em meio de tantos sentimentos que se apossam de cada um por exemplo, o desânimo e sobrecarga de atividades. No que diz respeito aos indicadores de depressão $(M=10.1, D P=7.40)$, avaliados através do PHQ-9 $(\alpha=.92)$, a média indica que os professores transitam com níveis moderados de sintomas depressivos. Levando em consideração que os escores total variam de 0 a 27, a classificação dos níveis moderados se dá entre 10 e 14. 


\section{Tabela 2}

Estatísticos descritivos das variáveis tratadas neste estudo

\begin{tabular}{lccccc}
\hline Variáveis & $n$ & $M$ & $D P$ & Min & Max \\
\hline Percepção de Ameaça COVID-19 & 280 & 32.81 & 6.875 & 10 & 42 \\
Percepção Social de Ameaça ao Trabalho & 273 & 4.21 & .947 & 1.38 & 6.38 \\
Saúde Mental (QSG-12) & 275 & 28.23 & 7.687 & 11 & 44 \\
Depressão (PHQ-9) & 279 & 10.1 & 7.401 & 0 & 27 \\
Impacto do COVID-19 & 279 & 34.99 & 12.095 & 9 & 61 \\
Satisfação com a Vida & 279 & 22.35 & 6.58 & 5 & 35 \\
\hline
\end{tabular}

A saúde mental de maneira geral, nesta pesquisa apresentou uma consistência interna de $\alpha=.92$, avaliada pelo QSG-12 apresenta $M=28.23(D P=7.68)$, com o ponto de corte do sintomático vs. assintomático consiste em $3 / 4$ pontos, 52/53 pontos no sistema tipo Likert de 1 a 4, indicando que quanto menor o escore, melhor será a condição de saúde mental (Pasquali et al., 1994), que foi o utilizado nesta pesquisa com professores. Isto indica que a amostra pode ser considerada assintomática. Neste estudo, observa-se que embora os professores experimentem desconfortos, ainda que em contexto adverso, a saúde mental continua preservada.

\section{Relações entre Percepção de ameaça e QSG-12, PHQ-9, Impacto da COVID-19 e Satisfação com}

$a$ vida (onde não indicado de outra forma, para $r: \mathrm{gl}=279$ )

As análises encontraram que a percepção de ameaça ao COVID-19 $(\alpha=.81)$, tem uma relação significativa com a saúde mental avaliada pelo QSG-12: $r(275)=.40 ; p<.01$. Isto significa que os professores de alguma maneira se encontram afetados por insônia, agoniado(a), uma sensação de não superar as dificuldades, entre outros aspectos que a ameaça do COVID-19 proporciona. Outrossim, se observa a relação significativa da percepção de ameaça ao coronavírus, ainda que considerada baixa, com a depressão analisado pelo PHQ-9, na medida em que os professores têm a percepção ameaçante sobre o coronavírus, informam sentir-se desmotivados(as), deprimidos e sem perspectivas: $r=.33, p<.01$.

Consequentemente, a percepção de ameaça informada pelos docentes e o impacto do COVID-19 mostrou uma relação significativa positiva, como um fator que afeta suas vidas financeiras e/ou psicológica, dificuldade para trabalhar ou estudar, ou tomar conta das coisas em casa: $r=.34, p<.01$.

Diante de vivências em circunstâncias adversas como é um momento de pandemia, notadamente alguns domínios específicos da vida como saúde e família reverberam na satisfação com a vida ( $\alpha=.92$ ), como se observa na relação significativa, embora negativa, que 
quanto maior a percepção de ameaça por COVID-19, menor será a satisfação com a vida $r=-.26$, $p<.01$ demonstrados pelos professores, entretanto, eles(as) não percebem da mesma maneira a relação entre a percepção da ameaça ao trabalho e a satisfação com a vida $r=.15, p<.05$, ainda que seja uma correlação desprezível.

Relações entre Percepção social da ameaça ao Trabalho, Impacto da Covid-19, QSG-12 e PHQ-9

No que diz respeito a percepção social de ameaça ao trabalho e o impacto do COVID-19 constatou-se relação negativa: $r(272)=-.31, p<.01$. Ou seja, os docentes relatam sentirem-se ameaçados, amedrontados de não poder acudir as reuniões marcadas de última hora e não se sentir apoiados pelas coordenações.

Com relação a percepção social de ameaça ao trabalho e a saúde mental dos professores $(r(268)=-.46, p<.01)$, foi possível verificar, diante da complexidade que envolve uma pandemia, quer estando o professor (a) no seguimento público ou privado, que a saúde mental estará mais fragilizada e, em alguns casos, poderá intensificar este desconforto ou sofrimento psíquico. Neste mesmo sentido, houve relação negativa e fraca, entre a percepção social de ameaça ao trabalho com os sintomas depressivos $r(268)=-.35, p<.01$, em que as queixas relatadas pelos professores são concernentes a sentir pouco interesse ou pouco prazer em realizar as coisas, sentir-se cansados e com pouca energia nas últimas semanas.

Relações entre Saúde mental, Depressão, Impacto da Covid-19 e Satisfação com a vida (gl para $r=275)$

No quesito saúde mental, avaliada pelo QSG-12, os resultados encontrados foram significativos, indicando uma correlação com a depressão, notadamente que estes indicadores se tornam mais presentes no contexto pandêmico: $r=.78, p<.01$. As questões relacionadas na confiança em si mesmo, sentir-se pouco feliz e deprimidos, somado as capacidades para tomar decisões entre outras coisas se fragilizam nos professores. Igualmente poder-se-á dizer que existe uma relação positiva com respeito a saúde mental e ao impacto do COVID-19: $r=.59, p$ $<.01$. Isto significa, por exemplo, que os professores vêm experimentando resultados psicológicos adversos podendo ser na forma de esgotamento, ansiedade, medos e incertezas, possibilidades de transmitir ou ser infectado. Finalmente, a correlação entre a saúde mental e satisfação com a vida mostra que os professores sentem desconforto em sua saúde mental não desfrutando de suas atividades diárias, afetando a capacidade de concentração e nas condições de suas vidas e satisfação com a mesma: $r=-.47, p<.01$. A Tabela 3 oferece de maneira detalhada as correlações de todas as variáveis tratadas na pesquisa. 


\section{Tabela 3}

Correlação entre Percepção da Ameaça ao COVID-19, Percepção Social de Ameaça ao Trabalho, Saúde Mental, Depressão, Impacto do COVID-19 e Satisfação com a vida

\begin{tabular}{lccccc}
\hline & 1. PA & 2. PSAT & 3. SM & 4. D & 5. SV \\
\hline 1. Percepção de Ameaça COVID-19 & - & & & & \\
2. Percepção Social de Ameaça ao Trabalho & $.495^{* *}$ & - & & & \\
3. Saúde Mental (QSG-12) & $.403^{* *}$ & $-.460^{* *}$ & - & & \\
4. Depressão (PHQ-9) & $.331^{* *}$ & $-.351^{* *}$ & $.780^{* *}$ & - & - \\
5. Impacto do COVID-19 & $.337^{* *}$ & $-.313^{* *}$ & $.588^{* *}$ & $.498^{* *}$ & - \\
6. Satisfação com a Vida & $-.260^{* *}$ & $.172^{* *}$ & $-.465^{* *}$ & $-.469^{* *}$ & $-.521^{* *}$ \\
\hline
\end{tabular}

${ }^{* *} p<.01$,

No que diz respeito a apresentarem um maior nível de sintomas depressivos como consequência da pandemia COVID-19, foi possível observar quanto ao nível de gravidade avaliado pelo PHQ-9, que 29.4\% dos docentes encontram-se no nível transtorno depressivo leve. Aqui vale ressaltar, que majoritariamente a amostra é de professores do seguimento público, que teve o início do ano letivo entre o final do primeiro semestre e outros no início no segundo semestre de 2020 (Tabela 4).

\section{Tabela 4}

Distribuição das frequências de depressão dos professores quanto aos níveis de gravidade - PHQ$9(N=279)$

\begin{tabular}{|c|c|c|c|c|c|}
\hline & \multicolumn{5}{|c|}{ Níveis de gravidade do Transtorno Depressivo } \\
\hline & Mínimo & Leve & Moderado & $\begin{array}{c}\text { Moderadamente } \\
\text { Severo }\end{array}$ & Severo \\
\hline$f(\%)$ & $25.4(71)$ & $29.4(82)$ & $16.8(47)$ & $12.2(34)$ & $16.1(45)$ \\
\hline
\end{tabular}

Na Tabela 5 observa-se que os Estados da Bahia e Rio de Janeiro tiveram maior representatividade na prevalência dos sintomas depressivos analisados pelo PHQ-9. Nestes dois Estados, especialmente, que se situam no nível de classificação entre 10-14, sendo considerado como moderado. Os demais têm um número de participantes aquém do esperado, dificultando predizer sobre estes indicadores de sintomas depressivos.

\section{Tabela 5}

Escore médios dos Estados com pontuação total indicado pelo PHQ-9

\begin{tabular}{lccc|lccc}
\hline Estado & $n$ & $M$ & $D P$ & Estado & $n$ & $M$ & $D P$ \\
\hline Bahia & 56 & 10.00 & 7.811 & Minas Gerais & 4 & 10.50 & 9.747 \\
Ceará & 2 & 13.50 & 3.536 & Paraíba & 2 & 8.50 & 3.536 \\
Distrito Federal & 8 & 10.88 & 7.918 & Piauí & 2 & 7.00 & 2.828 \\
Espírito Santo & 2 & 13.50 & 19.092 & Rio de Janeiro & 191 & 10.14 & 7.353 \\
Goiás & 4 & 10.25 & 5.737 & São Paulo & 3 & 5.33 & 5.859 \\
\hline \multicolumn{7}{c}{ Total: $N=279 ; M=10.10 ; D P=7.401$} & \\
\hline
\end{tabular}




\section{Discussão}

O interesse primordial deste estudo foi conhecer e apresentar como os professores responderam a cognição social, representada pela percepção social, e a saúde mental durante a pandemia por COVID-19 em 2020. Para tal se discutiu a literatura em que foi possível observar escassez de acervo brasileiro sobre o tema, desde a divulgação mundial dos primeiros casos identificados em 2019 na China e no Brasil, abril de 2020, quando o Ministério da Saúde reportou a nação sobre os casos e óbitos. Assim sendo, em escalada crescente em todas as áreas do conhecimento vêm contribuindo para melhor entendimento das repercussões da crise sanitária causada pelo vírus SARS-CoV-2 e, nesse sentido, o estudo possibilitou constatar hipóteses e apresentar evidências que possam contribuir para literatura.

Nesta pesquisa, foi possível explorar sobre os impactos sofridos pelos professores nos diversos segmentos da educação brasileira. Durante a pandemia com relação ao sofrimento psíquico informado por indicadores depressivos e por meio da saúde mental em geral, verificaram-se níveis leves para moderado, dados que corroboram com estudos realizados com professores chineses em que se observou inúmeros docentes adoecidos mentalmente pela COVID-19, apresentando transtorno depressivo leve, transtorno afetivo bipolar, ansiedade generalizada, transtorno de adaptação e síndrome de burnout ou síndrome do esgotamento profissional (Wang \& Wang, 2020).

Com respeito a Cognição social dos professores participantes desta pesquisa, constatouse que quanto mais percepcionam a ameaça do COVID-19, menor satisfação com suas vidas. Este resultado se alinha aos estudos de Cacciopo e Hawkey sobre a percepção do isolamento social (2009), que afirmam que o isolamento é um fator de risco e pode contribuir para um pior desempenho cognitivo geral, um declínio cognitivo mais rápido, pior funcionamento executivo, mais negatividade e cognição depressiva, maior sensibilidade a ameaças sociais, um viés confirmatório na cognição social que é auto protetor e paradoxalmente autodestrutivo, antropomorfismo elevado e contágio que ameaça a coesão social. Este resultado sugere refletir sobre as condições de vida e o coronavírus em domínios que são importantes na vida das pessoas, como pensar que os recursos financeiros não fazem tanta diferença caso fiquem infectados; e para toda a humanidade os procedimentos de não haver resultados conclusivos e vacinas suficientes, até então, pode levar a esta percepção ameaçante.

Os resultados são similares aos estudos com delineamentos semelhantes em que a sobrecarga de trabalho e desmotivação dos professores, a dificuldade do uso das tecnologias 
por parte de alguns docentes gera angústia, tristeza e baixa autoestima em suas práticas diárias de ensino. Assim como a saúde emocional dos professores e suas fragilidades, confirmando as hipóteses levantadas na pesquisa, as quais são corroboradas com os estudos que vêm demonstrando que a pandemia COVID-19 pode impactar a saúde mental e o bem-estar psicológico associando-se a mudanças nas rotinas e nas relações familiares (Cluver et al., 2020; Ornell et al., 2020).

Por outro lado, o impacto do COVID-19 como um fator que afeta os professores na vida financeira, psicológica, dificuldade para trabalhar ou estudar, ou tomar conta das coisas em casa, se alinham as pesquisas Silva et al. (2020) que argumentam que às pressões sofridas pelos docentes relacionadas ao uso das tecnologias e ao trabalho acabam adoecendo.

Nota-se ainda que o estudo exploratório e correlacional ora apresentado oferta informações que sinalizam a necessidade de outras pesquisas com outros desenhos metodológicos, tais como estudos de coorte prospectiva ou longitudinais. Isso porque um acompanhamento aprofundado do público abordado, a partir do marco da pandemia e dos desfechos da mesma para a atuação docente, tende a evidenciar efeitos que, muito provavelmente, demarcarão um novo paradigma educacional dada a robustez do conhecimento que se alcançará para além observações e associações.

Através destes resultados é possível desenhar práticas e propostas interventivas para mitigar o possível sofrimento psíquico dos docentes em atividade remota no Brasil como consequência da pandemia. Inicialmente, seria interessante, levar em consideração as características sociais de cada contexto, os estudantes e as condições de trabalho dos professores. A formação de professores com o uso das ferramentas e plataformas digitais, de acordo com Rodrigues et al. (2015) se constitui em um recurso valioso para as práticas. Outrossim, intervenção psicossocial com os professores contemplando estratégias de coping, saúde mental, questões cognitivas e habilidades para tarefas usando e-learning (FelpetoGuerrero et al., 2015). Cabe aqui sinalizar as limitações da pesquisa, relacionadas primeiramente a amostra que em sua maioria está composta por mulheres e mães, seria interessante ampliar o estudo e que as diferenças de gênero na composição da amostra não fossem tão dessemelhantes. Por outra parte, a dificuldade de ter mais representatividade de outros Estados, o que seria importante ter uma ideia mais ajustada do cenário dos professores nas diversas regiões de maneira mais equilibrada. 


\section{Referências}

ANDERSON, Norman H. (Ed.) (1981). Foundations of information integration theory. Academic Press.

ARAÚJO, Francisco Jonathan O.; LIMA, Lígia S.A.; CIDADE, Pedro Ivo M.; NOBRE, Camila. B.; \& NETO, Modesto L. R. (2020). Impact of Sars-Cov-2 and its Reverberation in Global Higher Education and Mental Health. Psychiatry Research, 288, 112977. http://doi.org/10.1016/ j.psychres.2020.112977

CACIOPPO, Jonh T.; \& HAWKLEY, Louise C. (2009). Perceived social isolation and cognition. Trends in Cognitive Sciences, 13(10), 447-454. https://doi.org/10.1016/j.tics.2009.06.005 CACIOPPO, Jonh T.; \& PATRICK, William (2008). Loneliness: Human nature and the need for social connection. W. W. Norton; \& Company.

CACIOPPO, Jonh T.; HAWKLEY, Louise C.; ERNST, Jonh M.; BURLESON, Mary.; BERNTSON, Gary G.; NOURIANI, Bita; \& SPIEGEL, David (2006). Loneliness within a nomological net: An evolutionary perspective. Journal of Research in Personality, 40(6), 1054-1085. https://doi.org/10.1016/j.jrp.2005.11.007

CACIOPPO, Jonh T.; \& HAWKLEY, Louise C. (2005). People thinking about people: The vicious cycle of being a social outcast in one's own mind. In Williams, K.D., et al., (Eds.), The social outcast: Ostracism, social exclusion, rejection, and bullying (pp. 91-108). Psychology Press.

CLUVER, Lucie; LACHMAN, Jamie M.; SHERR, Lorraine; WESSELS, Inge., KRUG, Etienne; RAKOTOMALALA, S.; BLIGHT, Stephen; HILLIS, Susan; BACHMAN, Gretchen; GREEN, Ohad; BUTCHART, Alex; TOMLINSON, Mark; WARD, Catherine L.; DOUBT, Jennifer; \& MCDONALD, Kerida (2020). Parenting in a time of COVID-19. The Lancet. https://doi.org/10.1016/\$0140-6736(20)30736-4

CONWAY, Lucian G. III; WOODARD, Shailee R.; \& ZUBROD, Alivia (2020, Abril 7). Social Psychological Measurements of COVID-19: Coronavirus Perceived Threat, Government Response, Impacts, and Experiences Questionnaires. https://doi.org/10.31234/osf.io/z2x9a

DIENER, Ed; EMMONS, Robert A.; LARSEN, Randy; \& GRIFFIN, Sharon (1985). The Satisfaction with Life Scale. Journal of Personality Assessment, 49, 71-5. https://doi.org/10.1207/ s15327752jpa4901_13

DUBEY, Souvik.; BISWAS Payel.; GHOSH, Ritwik.; CHATTERJEE, Subhankar; DUBEY, Muhua J.; CHATTERJEE, Subham; LAHIRI, Durjoy; \& LAVIE, Carl J. (2020). Psychosocial impact of COVID-19. Diabetes; \& Metabolic Syndrome, 14(5), 779-788. https://doi.org/10.1016/j. dsx.2020.05.035 
FELPETO-GUERRERO, A., REY-IGLESIA, R., FERNÁNDEZ-VÁZQUEZ, A.; \& GARROTE-YÁÑEZ, D. (2015). Uso de plataformas e-learning y alfabetización digital en formación profesional a distancia. Revista de estudios e Investigación en Psicología y Educación, Vol. Extr.(13), A13-164. https://doi.org/10.17979/reipe.2015.0.13.490

FISKE, Susan T.; \& TAYLOR, Shelley E. (2016). Social cognition: From brains to culture. (3rd ed.). Sage.

FLETCHER, Richard; KALOGEROPOULOS, Antonis; \& NIELSEN, Rasmus K. (2020). News avoidance in the UK remains high as lockdown restrictions are eased. Reuters Institute: https://reuters institute.politics.ox.ac.uk/news-avoidance-uk-remains-high-lockdown-restrictions-are-eased GOUVEIA, Valdiney V.; GENARO, Alves; OLIVEIRA, Edson; \& BRANDÃO, Mauro (2005). Medindo a satisfação com a vida dos médicos no Brasil. Jornal Brasileiro de Psiquiatria, 54(4), 298305. Disponível em: https://www.researchgate.net/publication/279194945_Medindo_a_ satisfacao_com_a_vida_dos_medicos_no_Brasil

GOUVEIA, Valdiney V.; CHAVES, Sandra Souza da S.; OLIVEIRA, Isabel Cristina Possatti; DIAS, Mardonio Rique; GOUVEIA, Rildésia S. V.; \& ANDRADE, Palloma R. (2003). A utilização do QSG-12 na população geral: estudo de sua validade de construto. Psicologia: Teoria e Pesquisa, 19(3), 241-248. https://doi.org/10.1590/\$0102-37722003000300006

HAUBRICH, Deise B.; \& FROEHLICH, Cristiane (2020). Benefícios e desafios do Home Office em empresas de Tecnologia da Informação. Revista Gestão; \& Conexões, 9 (1), 167-184. https://doi.org/10.13071/regec.2317-5087.2020.9.1.27901.167-184

KROENKE, Kurt; SPITZER, Robert L.; \& WILLIAMS, Janet B. (2001). The PHQ-9: validity of a brief depression severity measure. Journal of General Internal Medicine, 16, 606-13. https://doi.org/10.1016/S0140-6736(20)30460-8

LAI, Keke; \& GREEN, Samuel B. (2016). The problem with having two watches: Assessment of fit when RMSEA and CFI disagree. Multivariate Behavioral Research, 51(2-3), 220-239. https://doi.org/10.1080/00273171.2015.1134306

LEYENS, Jacques-Phillippe; \& DARDENNE, Benoit (1996). Basic concepts and approaches in social cognition. In Miles Hewstone, Wolfgang Stroebe; \& Geoffrey Stephenson (Eds.), Introduction to Social Psychology (pp. 109-134). Blackwell.

LIEBERMAN, Matthew D.; \& PFEIFER, Jennifer H. (2005). The self and social perception: Three kinds of questions in social cognitive neuroscience. In Alexander Easton \& Nathan Emery (Eds.), The cognitive neuroscience of social behavior (pp. 195-235). Psychology Press. Disponível em: https://doi.org/10.4324/9780203311875_chapter_7 
MCKIMM, Judy; GIBBS, Trevor; BISHOP, Joanna E.; \& JONES, Paul (2020). Health Professions' Educators' Adaptation to Rapidly Changing Circumstances: The Ottawa 2020 Conference Experience. MedEdPublish, 1(9)1-8. https://research.bond.edu.au/en/publications/healthprofessions-educators-adaptation-to-rapidly-changing-circu

MUÑOZ, Rafael (2020, abril 8). A Experiência internacional com os impactos da COVID-19 na educação. Nações Unidas Brasil. https://brasil.un.org/pt-br/85481-artigo-experienciainternacional-com-os-impactos-da-covid-19-na-educacao

MURRAY, Sandra L.; BELLAVIA, Gina M.; \& ROSE, Paul E. (2003). Once hurt, twice hurtful: How perceived regard regulates daily marital interactions. Journal of Personality and Social Psychology, 84(1)126-147. https://doi.org/10.1037/0022-3514.84.1.126

ORNELL, Felipe; SCHUCH, Jaqueline B.; Sordi, Anne; \& KESSLER, Felix H. P. (2020). "Pandemic fear" and COVID-19: Mental health burden and strategies. Brazilian Journal of Psychiatry. 42(3), 232-235. https://doi.org/10.1590/1516-4446-2020-0008

OROZCO-SOLÍS, M.G.; BRAVO-ANDRADE, H. R.; RUVALCABA-ROMERO, N. A.; \& ALFAROBERACOECHEA, L. N. (2021). Teen resilience: the role of school and family connectedness. Revista de estudios e Investigación en Psicología y Educación, 8, (1), 20-36. https://doi. org/10.17979/reipe.2021.8.1.7097

ORTIZ, Phillip A. (2020). Teaching in the time of COVID-19. Biochemistry and molecular biology education: a bimonthly publication of the International Union of Biochemistry and Molecular Biology. Editorial. http://doi.wiley.com/10.1002/bmb.21348

OSTROM, Thomas M. (1984). The sovereignty of social cognition. In Robert Wyer; \& Thomas Srull (Eds.), Handbook of Social Cognition (Vol. 1, pp. 1-38). Erlbaum.

PASQUALI, Luiz; GOUVEIA, Valdiney; BANDEIRA, Wagner A.; MIRANDA, Fábio; \& RAMOS, André L. (1996). Questionário de Saúde Geral de Goldenberg QSG: adaptação brasileira. Psicologia: Teoria e Pesquisa, 16(3), 421-437. Disponível em: https://www.researchgate. net/publication/279191898_Questionario_de_Saude_Geral_de_Goldberg_QSG_Adapta cao_Brasileira

REIS, Nélio; OLIVEIRA, Cristina C.; \& ANDRADE, Alequexandre (2020). Covid-19 e o calendário escolar brasileiro: medo e frustração. Revista Inovação Social, 2(1), 52-68. Disponível em: https://www.revistainovacaosocial.com.br/edi\%C3\%A7\%C3\%B5es-anteriores/vol-2-n1jan-a-abr-2020

RODRIGUES, Alessandra; REZENDE-Junior, Mikael Frank; STANO, Rita de Cássia M. T. (2015). Recursos digitais na formação de professores de Ciências: alargamento de sentidos. 
Revista de estudios e Investigación en Psicología y Educación, Extr.(6), A6-048. https://doi. org/10.17979/reipe.2015.0.06.165

ROMERO-CANYAS, Rainer; \& DOWNEY, Geraldine D. (2005). Rejection sensitivity as a predictor of affective and behavioral responses to interpersonal stress: A defensive motivational system. In Kipling D. Williams, Joseph P. Forgas, \& William Von Hippel (Eds.), The social outcast: Ostracism, social exclusion, rejection, and bullying (pp. 131-154). The Psychology Press.

SANTOS, Iná S.; TAVARES, Beatriz F.; MUNHOZ, Tiago N.; PIO DE ALMEIDA, Laura S.; BARRETO, Nathalia T. da Silva, et al. (2013). Sensibilidade e especificidade do Patient Health Questionnaire-9 (PHQ-9) entre adultos da população geral. Cadernos de Saúde Pública, 29(8),1533-1543. http://doi.org/10.1590/0102-311X00144612

SARRIERA, Jorge C.; SCHWARCZ, C.; \& CÂMARA, Sheila G. (1996). Bem-estar psicológico: análise fatorial da escala de Goldberg (GHQ-12) numa amostra de jovens. Psicologia Reflexão e Crítica, 9, 293-306. Disponível em: https://www.researchgate.net/publication/2752764 58_Bem-Estar_Psicologico_Analise_Fatorial_da_Escala_Golberg_Ghq-12_Numa_Amostra _de_Jovens

SCHREIBER, James B.; STAGE, Francis K.; KING, Jamie, NORA, Amaury; \& BARLOW, Elizabeth A. (2006). Reporting structural equation modeling and confirmatory factor analysis results: A review. The Journal of Educational Research, 99(6), 323-337. https://doi.org/10.3200/ JOER.99.6.323-338

SHAW, Keith (2020, Abril 2). Colleges expand VPN capacity, conferencing to answer COVID-19. NETWORKWORD: https://www.networkworld.com/article/3535415/colleges-expand-vpncapacity-conferencing-to-answer-covid-19.html

SILVA, Andrey F.; ESTRELA, Fernanda M.; LIMA, Nayara S.; \& ABREU, Carlos T. de A (2020). Saúde mental de docentes universitários em tempos de pandemia Physis: Revista de Saúde Coletiva, 30(2), 1-4. https://doi.org/10.1590/s0103-73312020300216

SPITZER, Robert L.; KROENKE, Kurt; \& WILLIAMS, Janet B. (1999). Validation and utility of a selfreport version of PRIME-MD: the PHQ primary care study. Primary Care Evaluation of Mental Disorders. Patient Health Questionnaire. JAMA. 10, 282(18):1737-44. https://doi. org/10.1001/jama.282.18.1737

TAYLOR, Steven (2017). Clinician's guide to PTSD. A Cognitive Behavior Approach (2nd ed.). Guildford.

TAYLOR, Steven (2019). Anxiety sensitivity. In J. S. Abramowitz; \& S. M. Blakey (Eds.), Clinical handbook of fear and anxiety: Psychological processes and treatment mechanisms (pp. 65-80). American Psychological Association. https://doi.org/10.1037/0000150-004 
VIEIRA, Joana B.; PIERZCHAJLO, Stephen; JANGARD, Simon; MARSH, Abigail A.; \& OLSSON, Andreas (2020). Perceived threat and acute anxiety predict increased everyday altruism during the COVID-19 pandemic. PsyArXiv Preprints. https://doi.org/10.31234/osf.io/n3t5c WANG, Jia; \& WANG, Zhifeng (2020). Strengths, weaknesses, opportunities and threats (SWOT) Analysis of China's prevention and control strategy for the COVID-19 epidemic. International Journal of Environmental Research and Public Health, 17(7), 2235. https://doi.org/10.3390/ijerph17072235

WEISS, Robert S. (1973). Loneliness: The experience of emotional and social isolation. MIT Press. WHEATON, Michael G.; ABRAMOWITZ, Jonathan S.; BERMAN, Noah; FABRICANT, Laura E.; \& OLATUNJI, O. (2012). Psychological predictors of anxiety in response to the HINI (swine flu) pandemic. Cognitive Therapy and Research, 36(3), 210-218. https://doi.org/10.1007/ s10608-011-9353-3

ZHONG, Chen-Bo; \& LEONARDELLI, Geoffrey J. (2008). Cold and lonely: does social exclusion literally feel cold? Psychology Science, 19(9), 838-842. https://doi.org/10.1111/j.14679280.2008.02165.x

Fecha de recepción: 6 de septiembre de 2021.

Fecha de revisión: 15 de noviembre de 2021. Fecha de aceptación: 20 de noviembre de 2021. Fecha de publicación: 1 de diciembre de 2021. 